\title{
Adiabatic theorem for non-hermitian time-dependent open systems
}

\author{
Avner Fleischer and Nimrod Moiseyev
}

July 5, 2018

Department of Chemistry and Minerva Center of Nonlinear Physics in Complex Systems Technion - Israel Institute of Technology Haifa 32000, Israel.

\begin{abstract}
In the conventional quantum mechanics (i.e., hermitian QM) the adiabatic theorem for systems subjected to time periodic fields holds only for bound systems and not for open ones (where ionization and dissociation take place) [D. W. Hone, R. Ketzmerik, and W. Kohn, Phys. Rev. A 56, 4045 (1997)]. Here with the help of the (t,t') formalism combined with the complex scaling method we derive an adiabatic theorem for open systems and provide an analytical criteria for the validity of the adiabatic limit. The use of the complex scaling transformation plays a key role in our derivation. As a numerical example we apply the adiabatic theorem we derived to a 1D model Hamiltonian of Xe atom which interacts with strong, monochromatic sine-square laser pulses. We show that the generation of odd-order harmonics and the absence of hyper-Raman lines, even when the pulses are extremely short, can be explained with the help of the adiabatic theorem we derived.
\end{abstract}

03.65.-w, 42.50.Hz, 42.65.-Ky, 32.80.Rm

\section{Motivation}

When matter is exposed to intense laser fields, high harmonics (HHs) of the incident radiation may be produced. Usually, only odd harmonics are obtained even when the laser pulses are short (for theoretical and experimental work which demonstrates this see [1] and 2], respectively). Since the duration of the pulse in time is inversely proportional to its width in energy space, one may find this result surprising, as one may expect to obtain also a large distribution of frequencies in the scattered field. Why are only odd harmonics obtained even when the laser pulses are short?

For CW lasers (and symmetric field-free potential) using the non-hermitian Floquet theory it was proved that only odd harmonics are obtained when the 
dynamics is controlled by a single resonance Floquet quasienergy (QE) state [3], 4]. When laser pulses are used it was argued that this proof holds since usually the populated resonance states are associated with very different lifetimes and the dynamics is controlled by the resonance state which has the longest lifetime. However, this argument may hold only when the duration of the laser pulses is large enough to enable the decay of the short lived resonances. Indeed numerical simulations showed that the harmonic generation spectra (HGS) as obtained from a single non-hermitian (complex scaled) resonance Floquet state is in a remarkable agreement with the results obtained from conventional (i.e., hermitian) time dependent simulation [5].

The question that is addressed in this work is weather an analytical criteria for the shape and duration of the laser pulse for which the system is controlled by a single resonance Floquet state can be given. It is obvious that the question regarding the possibility of population of a single resonance state is connected with the question regarding the degree of adiabaticity of the process. The question is therefore under which conditions can a short laser pulse be defined as adiabatic one. The answer to this question is important not only to harmonic generation (HG) studies but also for other, more general studies where lasers are used to control the dynamics, for example adiabatic STIRAP procedures [6].

In order to answer this question we use the $\left(\mathrm{t}, \mathrm{t}^{\prime}\right)$ formalism [7] together with the non-hermitian quantum mechanics (NHQM) formalism. The use of NHQM formalism to describe the dynamics of atoms/molecules subjected to $\mathrm{CW}$ laser fields is essential, since only then can the dynamics be described in terms physical, square integrable, resonance Floquet states. Otherwise, the description of the dynamics in terms of hermitian Floquet states results in very little physical insight on the problem, as well as numerical problems, not to mention that it is limited to the description of bound systems only. In the hermitian case the spectrum is continuous and becomes discrete only due to the use of finite box quantization; moreover, a single Floquet state in hermitian QM can not describe neither the resonance phenomena nor the field ionization phenomena [8].

Our strategy is as follows. In section 2 we give a brief review of the formalism used in our derivation [namely the $\left(t, t^{\prime}\right)$ formalism for hermitian and non-hermitian Hamiltonians]. In section 3 we introduce the new derivation of the adiabatic theorem for open quantum systems in strong laser pulses. In section 4 we apply the adiabatic theorem as derived in section 3 to a test-case model hamiltonian which describes a 1D Xe atom subjected to a sin-square pulse of monochromatic laser. In section 4 we conclude.

\section{A brief review of the $\left(t, t^{\prime}\right)$ formalism}

The $\left(t, t^{\prime}\right)$ formalism enables one to obtain analytical solutions for any timedependent Schrödinger equation (TDSE) with time-dependent Hamiltonians. The formalism rests on lifting of the TDSE to an extended Hilbert space, prop- 
agation of the wavefunction there and finally projecting back to the physical Hilbert space.

The solution of a general TDSE is given as usual by:

$$
H(\mathbf{r}, t) \Psi(\mathbf{r}, t)=i \hbar \frac{\partial}{\partial t} \Psi(\mathbf{r}, t)
$$

with the initial condition $\Psi(\mathbf{r}, t=0)$ and the vector operator $\mathbf{r}$ describes the internal degrees of freedom. It has been shown by Peskin and Moiseyev that by regarding time as an extra coordinate $t^{\prime}$, one can obtain another Schrödinger equation with a time independent Hamiltonian in the extended Hilbert space $\left(\mathbf{r}, t^{\prime}\right)$, whose solution $\bar{\Psi}\left(\mathbf{r}, t^{\prime}, t\right)$ has an analytical time dependence (given by the analytical time evolution operator associated with time-independent Hamiltonians) [7. Our desired wavfunction $\Psi(\mathbf{r}, t)$ can be deduced from this wavfunction by a simple operation (that will be shown later). The advantage is that efficient propagation schemes designed for solving the TDSE with time-independent Hamiltonians could then be used also for time dependent Hamiltonians, thus releasing one from the difficulties associated with time ordering.

Let us define the following Floquet-type operator

$$
\left.H_{F}\left(\mathbf{r}, t^{\prime}\right) \equiv\left(H(\mathbf{r}, t)-i \hbar \frac{\partial}{\partial t}\right)\right|_{t^{\prime}=t}=H\left(\mathbf{r}, t^{\prime}\right)-i \hbar \frac{\partial}{\partial t^{\prime}}, 0 \leq t^{\prime} \leq t_{f}
$$

where $t^{\prime}$ should be regarded as a coordinate and $t_{f}$ is the final time of propagation. When the Hamiltonian $H(\mathbf{r}, t)$ is time periodic with period $T$, the operator is the Floquet operator and $t_{f} \equiv T$. Provided that correct boundary conditions are chosen for the $t^{\prime}$ coordinate, this operator is hermitian since it is the sum of two hermitian operators. This operator has eigenstates and eigenvalues which are given by the eigenvalue equation:

$$
H_{F}\left(\mathbf{r}, t^{\prime}\right) \phi_{\alpha}\left(\mathbf{r}, t^{\prime}\right)=\varepsilon_{\alpha} \phi_{\alpha}\left(\mathbf{r}, t^{\prime}\right)
$$

and the set of eigenstates is complete in the extended Hilbert space $\left(\mathbf{r}, t^{\prime}\right)$ with respect to the inner product

$$
\left\langle\left\langle\phi_{\alpha} \mid \phi_{\alpha^{\prime}}\right\rangle\right\rangle_{\mathbf{r}, t^{\prime}} \equiv \frac{1}{t_{f}} \int_{0}^{T_{p}} d t^{\prime} \int_{-\infty}^{\infty} d^{3} r \phi_{\alpha}^{*}\left(\mathbf{r}, t^{\prime}\right) \phi_{\alpha^{\prime}}\left(\mathbf{r}, t^{\prime}\right)=\delta_{\alpha, \alpha^{\prime}}
$$

Say the following TDSE with time-independent Hamiltonian need to be solved with the initial state $\bar{\Psi}\left(\mathbf{r}, t^{\prime}, t=0\right)$ :

$$
H_{F}\left(\mathbf{r}, t^{\prime}\right) \bar{\Psi}\left(\mathbf{r}, t^{\prime}, t\right)=i \hbar \frac{\partial}{\partial t} \bar{\Psi}\left(\mathbf{r}, t^{\prime}, t\right)
$$

Using the definition of $H_{F}\left(\mathbf{r}, t^{\prime}\right)$ this equation reads

$$
H\left(\mathbf{r}, t^{\prime}\right) \bar{\Psi}\left(\mathbf{r}, t^{\prime}, t\right)=i \hbar\left(\frac{\partial}{\partial t}+\frac{\partial}{\partial t^{\prime}}\right) \bar{\Psi}\left(\mathbf{r}, t^{\prime}, t\right)
$$

By setting the cut $t=t^{\prime}$ on $\mathrm{Eq}[6$ one gets: 


$$
\left.\left[H\left(\mathbf{r}, t^{\prime}\right) \bar{\Psi}\left(\mathbf{r}, t^{\prime}, t\right)\right]\right|_{t^{\prime}=t}=H(\mathbf{r}, t)\left[\left.\bar{\Psi}\left(\mathbf{r}, t^{\prime}, t\right)\right|_{t^{\prime}=t}\right]=i \hbar \frac{\partial}{\partial t}\left[\left.\bar{\Psi}\left(\mathbf{r}, t^{\prime}, t\right)\right|_{t^{\prime}=t}\right]
$$

where the following property

$$
\frac{\partial}{\partial t}\left[\left.\Psi\left(\mathbf{r}, t^{\prime}, t\right)\right|_{t^{\prime}=t}\right]=\left.\left[\left(\frac{\partial}{\partial t}+\frac{\partial}{\partial t^{\prime}}\right) \bar{\Psi}\left(\mathbf{r}, t^{\prime}, t\right)\right]\right|_{t^{\prime}=t}
$$

(which holds true for any function of $t^{\prime}$ and $t$. The relation between the solution of $\mathrm{Eq}[\mathrm{l}$ and the solution of the original TDSE (Eq 1 is given by

$$
\Psi(\mathbf{r}, t)=\left.\bar{\Psi}\left(\mathbf{r}, t^{\prime}, t\right)\right|_{t^{\prime}=t}
$$

provided that these two differential equations have the same initial condition. Hence,

$$
\left.\bar{\Psi}\left(\mathbf{r}, t^{\prime}, t\right)\right|_{t^{\prime}=t=0}=\Psi(\mathbf{r}, t=0)
$$

It is seen from Eq10 that apparently the initial condition isn't unique. While this holds true in case that one is interested only in the physical wavefunction $\Psi(\mathbf{r}, t)$, it should be noted that if one wishes to calculate physical quantities in the extended Hilbert space using the function $\bar{\Psi}\left(\mathbf{r}, t^{\prime}, t\right)$ and then go back to the original Hilbert space, and get the correct results, the initial condition in the extended Hilbert space should behaves as a delta-function in $t^{\prime}$. The correct initial condition will therefore be $\bar{\Psi}\left(\mathbf{r}, t^{\prime}, t=0\right)=\Psi(\mathbf{r}, t=0) \delta\left(t^{\prime}\right)$.

The main advantage of the $\left(t, t^{\prime}\right)$ formalism from a numerical point of view is that it enables the use of an analytical expression for the time evolution operator, without the necessity of time ordering, even when the Hamiltonian is strongly time-dependent. Any TDSE with time-dependent Hamiltonian could be replaced by a different TDSE, with time-independent Hamiltonian, for which a greater number of accurate integration schemes exist and the solution is given formally by $\left.\bar{\Psi}\left(\mathbf{r}, t^{\prime}, t\right)=e^{-\frac{i}{\hbar} H_{F}\left(\mathbf{r}, t^{\prime}\right) t} \bar{\Psi}\left(\mathbf{r}, t^{\prime}, t=0\right)\right)$. The price one pays however is that the new TDSE need to be integrated over one more dimension.

The main advantage of the $\left(t, t^{\prime}\right)$ formalism from a conceptional point of view is that it enables to describe any time-dependent dynamics in terms of stationary eigenstates and eigenvalues.

So far the derivation has been carried out within the framework of the conventional (i.e., hermitian) quantum mechanics. Using the complex-scaling (CS) transformation 9, 10]

$$
H_{F}\left(\mathbf{r}, t^{\prime}\right) \longrightarrow H_{F}^{\theta}\left(\mathbf{r}, t^{\prime}\right) \equiv H\left(\mathbf{r} e^{i \theta}, t\right)-i \hbar \frac{\partial}{\partial t}
$$

the quasienergy spectrum of the Floquet Hamiltonian becomes complex and square-integrable resonance states, that were embedded in the continuum in the unscaled problem, are uncovered. For sake of simplicity we drop the index $\theta$ in all $\theta$-dependent expressions (operators, eigenvalues, eigenvectors etc.) in the proceeding text. 
The Floquet operator $H_{F}$ can be represented with the orthogonal Fourier basis set $\frac{1}{\sqrt{T}} e^{i \omega n t^{\prime}}, n=0, \pm 1, \pm 2, \ldots$ as a square matrix

$$
\left.\underline{\underline{\left[H_{F}(\mathbf{r})\right.}}\right]_{n^{\prime}, n}=\frac{1}{T} \int_{0}^{T} d t^{\prime} e^{-i \omega n^{\prime} t^{\prime}} H_{F}\left(\mathbf{r}, t^{\prime}\right) e^{-i \omega n t}
$$

where $T=2 \pi / \omega$. The left and right eigenvectors (bi-orthogonal set of eigenvectors, [11]) of this Floquet matrix are the Fourier components of the Floquet states as defined in $\mathrm{Eq}[$ that is

$$
\begin{aligned}
& {\left[\underline{\underline{H_{F}(\mathbf{r})}}\right]^{t} \vec{\varphi}_{\alpha}^{L}(\mathbf{r})=E_{\alpha} \vec{\varphi}_{\alpha}^{L}(\mathbf{r})} \\
& \left.\underline{\underline{\left[H_{F}(\mathbf{r})\right.}}\right] \vec{\varphi}_{\alpha}^{R}(\mathbf{r})=E_{\alpha} \vec{\varphi}_{\alpha}^{R}(\mathbf{r})
\end{aligned}
$$

where

$$
\left[\vec{\varphi}_{\alpha}^{L / R}(\mathbf{r})\right]_{n} \equiv \varphi_{\alpha, n}^{L / R}(\mathbf{r}) .
$$

Since in our case $\left[\underline{\underline{H_{F}(\mathbf{r})}}\right]^{t}=\left[\underline{\underline{H_{F}(\mathbf{r})}}\right]$ then $\vec{\varphi}_{\alpha}^{L}(\mathbf{r})=\vec{\varphi}_{\alpha}^{R}(\mathbf{r})$. There are two sets of eigenfunctions of the Floquet operator $H_{F}\left(\mathbf{r}, t^{\prime}\right)$

$$
\phi_{\alpha}^{R}\left(\mathbf{r}, t^{\prime}\right)=\sum_{n} \varphi_{\alpha, n}^{R}(\mathbf{r}) e^{i \omega n t^{\prime}}
$$

and

$$
\phi_{\alpha}^{L}\left(\mathbf{r}, t^{\prime}\right)=\sum_{n} \varphi_{\alpha, n}^{L}(\mathbf{r}) e^{-i \omega n t^{\prime}}=\sum_{n} \varphi_{\alpha, n}^{R}(\mathbf{r}) e^{-i \omega n t^{\prime}}
$$

As pointed out in [12] the c-product, which is associated with the non usual inner-product in linear algebra (see for example Wilkinson's text book [11]), reads

$$
\left(\phi_{\alpha}^{L}\left(\mathbf{r}, t^{\prime}\right) \mid \phi_{\alpha^{\prime}}^{R}\left(\mathbf{r}, t^{\prime}\right)\right)_{\mathbf{r}, t^{\prime}}=\sum_{n}\left(\varphi_{\alpha, n}^{R} \mid \varphi_{\alpha^{\prime}, n}^{R}\right)_{\mathbf{r}}=\delta_{\alpha, \alpha^{\prime}}
$$

where $\left(\phi_{\alpha}^{L}\left(\mathbf{r}, t^{\prime}\right) \mid \phi_{\alpha^{\prime}}^{R}\left(\mathbf{r}, t^{\prime}\right)\right)_{\mathbf{r}, t^{\prime}} \equiv\left\langle\phi_{\alpha}^{L *}\left(\mathbf{r}, t^{\prime}\right) \mid \phi_{\alpha^{\prime}}^{R}\left(\mathbf{r}, t^{\prime}\right)\right\rangle_{\mathbf{r}, t^{\prime}}$. However, as proposed recently by Moiseyev and Lein ([13]), the inner-product should be modified even further when time-dependent functions are used as basis set due to the time-asymmetry problem in NHQM. If we define $\phi_{\alpha}^{R}(\mathbf{r}, t)$ and $E_{\alpha}=E_{r_{\alpha}}-\frac{i}{2} \Gamma_{\alpha}$ to be the Floquet eigenfunctions and eigenvalues respectively, then the timedependent basis functions could be

$$
\Psi_{\alpha}^{R}\left(\mathbf{r}, t^{\prime}, t\right)=e^{-\frac{i}{\hbar} E_{\alpha} t} \phi_{\alpha}^{R}\left(\mathbf{r}, t^{\prime}\right)
$$

(which are solutions of the TDSE) and also the left functions

$$
\Psi_{\alpha}^{L}\left(\mathbf{r}, t^{\prime}, t\right)=e^{+\frac{i}{\hbar} E_{\alpha}^{*} t} \phi_{\alpha}^{L}\left(\mathbf{r}, t^{\prime}\right) .
$$


Following the modified definition of the inner product ("finite-range" product, "F-product" [13])

$$
\left(\Psi_{\alpha}^{L}(t) \mid \Psi_{\alpha^{\prime}}^{R}(t)\right)_{\mathbf{r}, t^{\prime}} \equiv e^{-\frac{i}{\hbar} E_{\alpha} t} e^{+\frac{i}{\hbar} E_{\alpha^{\prime}}^{*} t}\left(\phi_{\alpha}^{L}\left(\mathbf{r}, t^{\prime}\right) \mid \phi_{\alpha^{\prime}}^{R}\left(\mathbf{r}, t^{\prime}\right)\right)_{\mathbf{r}, t^{\prime}}=e^{-\Gamma_{\alpha} t} \delta_{\alpha, \alpha^{\prime}}
$$

it implies that the $\alpha^{\text {th }}$ quasienergy state decays exponentially in time. For more detailed discussion see 13, 14.

\section{The adiabatic theorem for time-dependent sys- tems}

The adiabatic theorem for time-dependent bound systems was derived in 1997 by Kohn et al. [15] and in 1999 by Holthaus et al. [16] who used the (t,t') formalism to describe the evolution of a system subjected to chirped laser pulses. As discussed by Kohn and co-workers, the adiabatic approach is not applicable for open systems since the quasienergy level spacing reduce to zero as the number of basis functions used in the numerical calculation is increased. To avoid this difficulty Baer et al. [17 applied the adiabatic theorem to timedependent open systems in the high frequency regime where the system was stabilized and the resonances (which were embedded in the continuum part of the Floquet spectra) became so narrow that they could be practically treated as bound states. The purpose of our work is to derive the adiabatic theorem for general time-dependent open systems where there are no bound states and the resonances aren't necessarily narrow. We are using the non-hermitian Floquet formalism (through the CS formalism) which allow us to describe the dynamics in term of non-hermitian resonance states (see for example 9]-21], and also the work of Day et al. 22] who used the NH Floquet multistate method to study the applicability of the single Floquet resonance approximation in the description of the dynamics of $\mathrm{H}$ atom subjected to intense laser fields of various strengths).

Below we derive the adiabatic theorem for time-dependent open systems using the extended $\left(\mathrm{t}, \mathrm{t}^{\prime}\right)$ formalism. By the term "extended" $\left(t, t^{\prime}\right)$ formalism we mean that in the same manner presented, one may add any number of time "coordinates" to the Schrödinger equation as one wishes, if by this a better understanding or easier solution of the problem is achieved. Here we found that by addition of 2 time "coordiantes" to the TDSE, we simplified the derivation of the adiabaticity criteria for photo-induced dynamical systems. In this sense, we are using a $\left(t, t^{\prime}, t^{\prime \prime}\right)$ formalism.

We would like to study the dynamics of a single active electron in an atom or molecule, subjected to a pulse of strong monochromatic linearly polarized laser

radiation. In the dipole approximation the TDSE which describes this process is:

$$
H(\mathbf{r}, t) \Psi^{R}(\mathbf{r}, t)=i \hbar \frac{\partial}{\partial t} \Psi^{R}(\mathbf{r}, t)
$$


where,

$$
H(\mathbf{r}, t)=H_{0}(\mathbf{r})-e \mathbf{r} \cdot \mathbf{f}(t) \cos (\omega t)
$$

and,

$$
\mathbf{f}(t) \equiv \varepsilon_{0} \mathbf{e}_{\mathbf{k}} f(t) .
$$

Here $f(t)$ is the function which describes the envelope of the laser pulse and $\mathbf{f}(t)$ is a vector as defined in Eq24 $\varepsilon_{0}$ is the laser's amplitude, $\mathbf{e}_{\mathbf{k}}$ is a unit vector in the direction of the electric component of the laser field, $\omega$ is the laser's frequency, with $T=2 \pi / \omega$ the optical period. $H_{0}(\mathbf{r})$ is the field-free Hamiltonian and the vector operator $\mathbf{r}$ describes the internal degrees of freedom (the coordinates are complex-scaled throughout) .

In the same spirit of subsection (2.1), we define the following operator

$$
H_{F}\left(\mathbf{r}, t^{\prime}, t^{\prime \prime}\right) \equiv \widetilde{H}\left(\mathbf{r}, t^{\prime}, t^{\prime \prime}\right)-i \hbar \frac{\partial}{\partial t^{\prime}}-i \hbar \frac{\partial}{\partial t^{\prime \prime}}
$$

where

$$
\widetilde{H}\left(\mathbf{r}, t^{\prime}, t^{\prime \prime}\right) \equiv H_{0}(\mathbf{r})-e \mathbf{r} \cdot \mathbf{f}\left(t^{\prime \prime}\right) \cos \left(\omega t^{\prime}\right)
$$

and $t^{\prime}, t^{\prime \prime}$ should be regarded as additional coordinates. Upon complex-scaling $23 H_{F}\left(\mathbf{r}, t^{\prime}, t^{\prime \prime}\right)$ becomes non-hermitian. Therefore the inner c-product should be used as mentioned before. The quasi-energy solutions of this complex-scaled Floquet-type operator are:

$$
\begin{aligned}
& H_{F}\left(\mathbf{r}, t^{\prime}, t^{\prime \prime}\right) \psi_{k}^{R}\left(\mathbf{r}, t^{\prime}, t^{\prime \prime}\right)=\lambda_{k} \psi_{k}^{R}\left(\mathbf{r}, t^{\prime}, t^{\prime \prime}\right) \\
& H_{F}^{\dagger *}\left(\mathbf{r}, t^{\prime}, t^{\prime \prime}\right) \psi_{k}^{L}\left(\mathbf{r}, t^{\prime}, t^{\prime \prime}\right)=\lambda_{k} \psi_{k}^{L}\left(\mathbf{r}, t^{\prime}, t^{\prime \prime}\right)
\end{aligned}
$$

where the symbol $H_{F}^{\dagger *}$ doesn't stand for an operator but for the transpose of the matrix representing the operator $H_{F}$. The eigenfunctions form a complete set in the extended Hilbert space $\mathbf{r}, t^{\prime}, t^{\prime \prime}$.

Say we want to solve the following TDSE with the initial state $\widetilde{\Psi}\left(\mathbf{r}, t^{\prime}, t^{\prime \prime}, t=\right.$ $0)$ :

$$
H_{F}\left(\mathbf{r}, t^{\prime}, t^{\prime \prime}\right) \widetilde{\Psi}^{R}\left(\mathbf{r}, t^{\prime}, t^{\prime \prime}, t\right)=i \hbar \frac{\partial}{\partial t} \widetilde{\Psi}^{R}\left(\mathbf{r}, t^{\prime}, t^{\prime \prime}, t\right)
$$

The solution to this equation is

$$
\widetilde{\Psi}^{R}\left(\mathbf{r}, t^{\prime}, t^{\prime \prime}, t\right)=e^{-\frac{i}{\hbar} H_{F}\left(\mathbf{r}, t^{\prime}, t^{\prime \prime}\right) t} \widetilde{\Psi}^{R}\left(\mathbf{r}, t^{\prime}, t^{\prime \prime}, t=0\right)=\sum_{k} c_{k} e^{-\frac{i}{\hbar} \lambda_{k} t} \psi_{k}^{R}\left(\mathbf{r}, t^{\prime}, t^{\prime \prime}\right)
$$

and a function $\widetilde{\Psi}^{L}\left(\mathbf{r}, t^{\prime}, t^{\prime \prime}, t\right)$, which is not a solution of any Schrödinger equation, is defined as 


$$
\widetilde{\Psi}^{L}\left(\mathbf{r}, t^{\prime}, t^{\prime \prime}, t\right)=\sum_{k} c_{k} e^{+\frac{i}{\hbar} \lambda_{k}^{*} t} \psi_{k}^{L}\left(\mathbf{r}, t^{\prime}, t^{\prime \prime}\right)
$$

where by taking the cut $t^{\prime}=t^{\prime \prime}=t=0$ on Eq 30 it is easily seen that the expansion coefficients are $c_{k}=\left(\left.\psi_{k}^{L}\left(\mathbf{r}, t^{\prime}, t^{\prime \prime}\right)\right|_{t^{\prime}=t^{\prime \prime}=0}\left|\widetilde{\Psi}^{R}\left(\mathbf{r}, t^{\prime}, t^{\prime \prime}, t\right)\right|_{t^{\prime}=t^{\prime \prime}=t=0}\right)_{\mathbf{r}}$. $\left[\left.e^{-\frac{i}{\hbar} \lambda_{k} t} \psi_{k}^{R}\left(\mathbf{r}, t^{\prime}, t^{\prime \prime}\right)\right|_{t^{\prime}=t^{\prime \prime}=t}\right.$ is a solution of the original TDSE (apply the cut $\mathrm{t}^{\prime}=\mathrm{t} "=\mathrm{t}$ on $\mathrm{Eq} 27$ and compare the result to the result obtained when the function $\left.e^{-\frac{i}{\hbar} \lambda_{k} t} \psi_{k}^{R}\left(\mathbf{r}, t^{\prime}, t^{\prime \prime}\right)\right|_{t^{\prime}=t^{\prime \prime}=t}$ is substituted in the original TDSE); therefore, any linear combination of these solutions is also a solution].

Let us now return to the main purpose of this article, the derivation of the adiabatic theorem for non-hermitian open systems. We would like to treat $t^{\prime \prime}$ as an adiabatic coordinate (this is the "coordinate" associated with the pulse envelope) in the same way that the electronic motion is separated from the nuclear one in the treatment of molecules within the Born-Oppenheimer approximation. First we define the following operator

$$
H_{a d}\left(\mathbf{r}, t^{\prime}, t^{\prime \prime}\right) \equiv \widetilde{H}\left(\mathbf{r}, t^{\prime}, t^{\prime \prime}\right)-i \hbar \frac{\partial}{\partial t^{\prime}}
$$

where $t^{\prime \prime}$ should be regarded as a parameter now. This means that this Hamiltonian is a Floquet Hamiltonian describing the interaction of the atom with $\mathbf{C W}$ laser of strength $\varepsilon_{1}$ where following Eq[24]

$$
\varepsilon_{1}=|\mathbf{f}(t)|=\varepsilon_{0} f(t)
$$

The eigenstates of this operator form a complete basis (in the $\mathbf{r}-t^{\prime}$ space), for every value of the parameter $t^{\prime \prime}$ :

$$
H_{a d}\left(\mathbf{r}, t^{\prime}, t^{\prime \prime}\right) \psi_{\alpha}^{a d, R}\left(\mathbf{r}, t^{\prime}, t^{\prime \prime}\right)=\varepsilon_{\alpha}^{a d}\left(t^{\prime \prime}\right) \psi_{\alpha}^{a d, R}\left(\mathbf{r}, t^{\prime}, t^{\prime \prime}\right) .
$$

Notice that due to the complex scaling, $\lambda_{k}$ and $\varepsilon_{\alpha}^{a d}\left(t^{\prime \prime}\right)$ get complex values.

We can expand each eigenstate of the complete problem (Eq 27) in this basis:

$$
\psi_{k}^{R}\left(\mathbf{r}, t^{\prime}, t^{\prime \prime}\right)=\sum_{\alpha^{\prime}} \psi_{\alpha^{\prime}}^{a d, R}\left(\mathbf{r}, t^{\prime}, t^{\prime \prime}\right) \chi_{\alpha^{\prime}, k}\left(t^{\prime \prime}\right)
$$

Substituting Eq 35 into Eq27 multiplying the obtained equation from the left hand side by $\psi_{\alpha^{\prime}}^{a d, L}\left(\mathbf{r}, t^{\prime}, t^{\prime \prime}\right)$ and integrating over $\mathbf{r}$ and $t^{\prime}$ one gets, in matrix notation, the equality:

$$
\left[-i \hbar \frac{\partial}{\partial t^{\prime \prime}} \underline{\underline{I}}+\left(\underline{\underline{E}}^{a d}\left(t^{\prime \prime}\right)+\underline{\underline{V}}\left(t^{\prime \prime}\right)\right)\right] \vec{\chi}_{k}\left(t^{\prime \prime}\right)=\lambda_{k} \vec{\chi}_{k}\left(t^{\prime \prime}\right)
$$

where

$$
\begin{aligned}
& {\left[\underline{\underline{E}}^{a d}\left(t^{\prime \prime}\right)\right]_{\alpha, \alpha^{\prime}}=\varepsilon_{\alpha}^{a d}\left(t^{\prime \prime}\right) \delta_{\alpha, \alpha^{\prime}},} \\
& \left.\left[\underline{\underline{V}}\left(t^{\prime \prime}\right)\right)\right]_{\alpha, \alpha^{\prime}}=\left(\left(\psi_{\alpha}^{a d, L}\left(\mathbf{r}, t^{\prime}, t^{\prime \prime}\right)\left|-i \hbar \frac{\partial}{\partial t^{\prime \prime}}\right| \psi_{\alpha^{\prime}}^{a d, R}\left(\mathbf{r}, t^{\prime}, t^{\prime \prime}\right)\right)\right)_{\mathbf{r}, t^{\prime}}
\end{aligned}
$$




$$
\left[\vec{\chi}_{k}\left(t^{\prime \prime}\right)\right]_{\alpha}=\chi_{\alpha, k}\left(t^{\prime \prime}\right)
$$

Notice that in case that the matrix on the left hand side of $\mathrm{Eq}[36$ is diagonal, a homogeneous systems of uncoupled equations is obtained. In such a case one should solve each equation separately. Therefore, the sum in Eq 35 reduces to a single product. This is exactly the adiabatic approximation as appears in the Born-Oppenheimer context. The next step in our derivation is to represent the matrix $\left.\underline{\underline{E}}^{a d}\left(t^{\prime \prime}\right)+\underline{\underline{V}}\left(t^{\prime \prime}\right)\right)$ by its spectral decomposition

$$
\begin{aligned}
& {\left[\underline{\underline{E}}^{a d}\left(t^{\prime \prime}\right)+\underline{\underline{V}}\left(t^{\prime \prime}\right)\right] \underline{\underline{D}}^{R}\left(t^{\prime \prime}\right)=\underline{\underline{D}}^{R}\left(t^{\prime \prime}\right) \underline{\underline{W}}\left(t^{\prime \prime}\right)} \\
& {\left[\underline{\underline{E}}^{a d}\left(t^{\prime \prime}\right)+\underline{\underline{V}}\left(t^{\prime \prime}\right)\right] \underline{\underline{D}}^{L}\left(t^{\prime \prime}\right)=\underline{\underline{D}}^{L}\left(t^{\prime \prime}\right) \underline{\underline{W}}\left(t^{\prime \prime}\right)}
\end{aligned}
$$

The matrix of eigenvalues $\underline{\underline{W}}\left(t^{\prime \prime}\right)$ is diagonal and the right and left eigenvectors are normalized with respect to each other in order to maintain the correct inner product:

$$
\left[\underline{\underline{D}}^{L}\left(t^{\prime \prime}\right)\right]^{t} \underline{\underline{D}}^{R}\left(t^{\prime \prime}\right)=\underline{\underline{I}}
$$

In the case that the matrix $\left(\underline{\underline{E}}^{a d}\left(t^{\prime \prime}\right)+\underline{\underline{V}}\left(t^{\prime \prime}\right)\right)$ is not strictly diagonal we can use first-order perturbation theory to get the first-order deviation from diagonal. If we treat the matrix $\underline{\underline{V}}\left(t^{\prime \prime}\right)$ as perturbation, we get

$$
\left[\underline{\underline{D}}^{R}\left(t^{\prime \prime}\right)\right]_{\alpha^{\prime}, \alpha}=\delta_{\alpha^{\prime}, \alpha}+\frac{\left[\underline{\underline{V}}\left(t^{\prime \prime}\right)\right]_{\alpha^{\prime}, \alpha}}{\varepsilon_{\alpha}^{a d}\left(t^{\prime \prime}\right)-\varepsilon_{\alpha^{\prime}}^{a d}\left(t^{\prime \prime}\right)}
$$

The matrix will be diagonal to a good approximation if

$$
A_{\alpha}\left(t^{\prime \prime}\right) \equiv \sum_{\alpha^{\prime} \neq \alpha}\left|\left[\underline{\underline{D}}^{R}\left(t^{\prime \prime}\right)\right]_{\alpha^{\prime}, \alpha}\right| \ll 1
$$

which produces the following adiabaticity criteria,

$$
A_{\alpha}\left(t^{\prime \prime}\right) \equiv \sum_{\alpha^{\prime} \neq \alpha}\left|\frac{\left(\left(\psi_{\alpha}^{a d}\left(\mathbf{r}, t^{\prime}, t^{\prime \prime}\right)\left|-i \hbar \frac{\partial}{\partial t^{\prime \prime}}\right| \psi_{\alpha^{\prime}}^{a d}\left(\mathbf{r}, t^{\prime}, t^{\prime \prime}\right)\right)\right)_{\mathbf{r}, t^{\prime}}}{\varepsilon_{\alpha^{\prime}}^{a d}\left(t^{\prime \prime}\right)-\varepsilon_{\alpha}^{a d}\left(t^{\prime \prime}\right)}\right| \ll 1
$$

Using the specific form of the Hamiltonian of the problem given in Eq23 and the Hellman-Feynman theorem one gets the adiabatic condition for timedependent open systems:

$$
A_{\alpha}\left(t^{\prime \prime}\right) \ll 1
$$

where

$$
A_{\alpha}\left(t^{\prime \prime}\right) \equiv|e| \hbar \varepsilon_{0}\left|\frac{d f\left(t^{\prime \prime}\right)}{d t^{\prime \prime}}\right| \sum_{\alpha^{\prime} \neq \alpha}\left|\frac{\left(\left(\psi_{\alpha}^{a d, L}\left(\mathbf{r}, t^{\prime}, t^{\prime \prime}\right)\left|\mathbf{r} \cdot \mathbf{e}_{\mathbf{k}} \cos \left(\omega t^{\prime}\right)\right| \psi_{\alpha^{\prime}}^{a d, R}\left(\mathbf{r}, t^{\prime}, t^{\prime \prime}\right)\right)\right)_{\mathbf{r}, t^{\prime}}}{\left(\varepsilon_{\alpha^{\prime}}^{a d}\left(t^{\prime \prime}\right)-\varepsilon_{\alpha}^{a d}\left(t^{\prime \prime}\right)\right)^{2}}\right|
$$


The index $\alpha$ is a super-index; since (Eq 34 it is easily seen that not only is $\psi_{\alpha}^{a d, L / R}\left(\mathbf{r}, t^{\prime}, t^{\prime \prime}\right)$ a solution of the eigenvalue equation, with eigenvalue $\varepsilon_{\alpha}^{a d}\left(t^{\prime \prime}\right)$, but also $e^{i \omega m t^{\prime}} \psi_{\alpha}^{a d, L / R}\left(\mathbf{r}, t^{\prime}, t^{\prime \prime}\right)$ is a solution, with the eigenvalue $\varepsilon_{\alpha}^{a d}\left(t^{\prime \prime}\right)+\hbar \omega m$, for any integer $m$. Let us take all the states whose corresponding eigenvalues lie in the interval $[0, \hbar \omega]$ (the first Brillouin zone) and define them to have index $m=0$; we call these states $\phi_{j}^{a d, L}\left(\mathbf{r}, t^{\prime}, t^{\prime \prime}\right), \phi_{j}^{a d, R}\left(\mathbf{r}, t^{\prime}, t^{\prime \prime}\right)$ and the corresponding eigenvalues $E_{j}^{a d}\left(t^{\prime \prime}\right)$ and get:

$$
\begin{gathered}
\psi_{\alpha}^{a d, R}\left(\mathbf{r}, t^{\prime}, t^{\prime \prime}\right) \equiv \phi_{j}^{a d, R}\left(\mathbf{r}, t^{\prime}, t^{\prime \prime}\right) e^{i \omega m t^{\prime}} \\
\psi_{\alpha}^{a d, L}\left(\mathbf{r}, t^{\prime}, t^{\prime \prime}\right) \equiv \phi_{j}^{a d, L}\left(\mathbf{r}, t^{\prime}, t^{\prime \prime}\right) e^{i \omega m t^{\prime}} \\
\varepsilon_{\alpha}^{a d}\left(t^{\prime \prime}\right) \equiv E_{j}^{a d}\left(t^{\prime \prime}\right)+\hbar \omega m
\end{gathered}
$$

where

$$
0 \leq E_{j}^{a d}\left(t^{\prime \prime}\right) \leq \hbar \omega
$$

Thus, the index $\alpha$ actually counts both the position of the quasienergy within the first Brillouin zone (the index $j$ ) and the Brillouin zone itself (the index $n$ ). With respect to the generalized inner product, two states with one or more of the indices $(j, n)$ different are orthogonal.

Going back to Eq45 now ,it is seen that the probability to couple an initial adiabatic state $\psi_{\alpha=(j, 0)}^{a d}\left(\mathbf{r}, t^{\prime}, t^{\prime \prime}\right)$ to any other adiabatic state $\psi_{\alpha^{\prime}=\left(j^{\prime}, m^{\prime}\right)}^{a d}\left(\mathbf{r}, t^{\prime}, t^{\prime \prime}\right)$ is given by,

$$
A_{(j, 0)}\left(t^{\prime \prime}\right)=F\left(t^{\prime \prime}\right) \cdot \sum_{j^{\prime} \neq j} a_{\left(j^{\prime}\right)}^{(j)}\left(t^{\prime \prime}\right)
$$

where

$$
\begin{gathered}
F\left(t^{\prime \prime}\right)=|e| \hbar \varepsilon_{0}\left|\frac{d f\left(t^{\prime \prime}\right)}{d t^{\prime \prime}}\right|, \\
a_{\left(j^{\prime}\right)}^{(j)}\left(t^{\prime \prime}\right)=\sum_{m \neq 0}\left|c_{\left(j^{\prime}, m\right)}^{(j, 0)}\left(t^{\prime \prime}\right)\right|
\end{gathered}
$$

and the functions $c_{\left(j^{\prime}, m\right)}^{(j, 0)}\left(t^{\prime \prime}\right)$, that will be termed as "adiabatic cross terms" from now on, are given by

$$
c_{\left(j^{\prime}, m\right)}^{(j, 0)}\left(t^{\prime \prime}\right)=\frac{\left(\left(\phi_{j}^{a d, L}\left(\mathbf{r}, t^{\prime}, t^{\prime \prime}\right)\left|\mathbf{r} \cdot \mathbf{e}_{\mathbf{k}} \cos \left(\omega t^{\prime}\right) e^{i \omega m t^{\prime}}\right| \phi_{j^{\prime}}^{a d, R}\left(\mathbf{r}, t^{\prime}, t^{\prime \prime}\right)\right)\right)_{\mathbf{r}, t^{\prime}}}{\left(E_{j^{\prime}}^{a d}\left(t^{\prime \prime}\right)-E_{j}^{a d}\left(t^{\prime \prime}\right)+\hbar \omega m\right)^{2}}
$$

Since the energies $E_{j^{\prime}}^{a d}\left(t^{\prime \prime}\right)$ are complex (the Hamiltonian is non-hermitian) then for $j \neq j^{\prime}$ it is most unlikely that $E_{j^{\prime}}^{a d}=E_{j}^{a d}$. It is clear that the denominator hardly ever vanishes even when $m=0$. This holds true even when $j=j^{\prime}$ but $m \neq 0$. 
Therefore, the criteria for a pulse to be considered adiabatic is that the condition

$$
A_{(j, 0)}\left(t^{\prime \prime}\right) \ll 1
$$

be fulfilled.

Who is the adiabatic state $\psi_{\alpha=(j, 0)}^{a d}\left(\mathbf{r}, t^{\prime}, t^{\prime \prime}\right)$ whose couplings to all other states $\psi_{\alpha^{\prime}=\left(j^{\prime}, m^{\prime}\right)}^{a d}\left(\mathbf{r}, t^{\prime}, t^{\prime \prime}\right)$ should remain small in the adiabatic limit? Assuming that the system is in a stationary state of the field-free problem $\varphi_{j}(\mathbf{r})$ before the action of the field (relaying on the superposition principle of the solutions of the TDSE generality is not lost by this assumption) and provided that the field is switched adiabatically, this Floquet resonanace state $\psi_{\alpha=(j, 0)}^{a d}\left(\mathbf{r}, t^{\prime}, t^{\prime \prime}\right)$ is the state which is "born" from the stationary state $\varphi_{j}(\mathbf{r})$ as the field is switched on. If the process is not done adiabatically many Floquet resonanace states will be populated, resulting in considerable couplings of $\psi_{\alpha=(j, 0)}^{a d}\left(\mathbf{r}, t^{\prime}, t^{\prime \prime}\right)$ to them and collapse of the adiabatic condition in Eq 54 The only adiabatic check which is physically meaningful is one in which $\alpha$ denotes a resonance state (which is associated with square-integrable function). $\alpha^{\prime}$ however stands for both resonances and rotated continuum states.

One should notice that the derivation of the adiabatic theorem presented above holds for many electron systems. In order to avoid complicated notation the symbol $\mathbf{r}$ can stand for many electrons. It also holds for polychromatic radiation, where the $\mathrm{CW}$ field is a collection of monochromatic fields with frequencies $\omega_{i}$ and phases $\varphi_{i}$.

Notice that for a given problem (given spectral profile of the CW field and given field-free potential), the sum over absolute value of the adiabatic cross terms should be calculated as function of the effective CW-field intensity (which is symbolized here through $t^{\prime \prime}$ ) only once. The adiabatic cross terms should then be converted to be functions of time, through the explicit time dependence of the pulse envelope and then the sum of their absolute values should be multiplied by the time derivative of the pulse envelope and by the maximal field intensity to obtain the final expression which indicates whether the adiabatic criteria is fulfilled or not.

It is easily seen in Eq 51 that for a given system, the shape and intensity of the laser pulse determines its adiabaticity since these parameters influence the shape-derivative term. A short pulse which is switched on or off abruptly and has a high maximal intensity, will most likely not be adiabatic.

In the case that the adiabaticity criteria is fulfilled, the sum in Eq 35 could be reduced to a single product:

$$
\psi_{k, j}^{R}\left(\mathbf{r}, t^{\prime}, t^{\prime \prime}\right) \cong \phi_{j}^{a d, R}\left(\mathbf{r}, t^{\prime}, t^{\prime \prime}\right) \chi_{j, k}\left(t^{\prime \prime}\right)
$$

and the adiabatic states are assigned with two good quantum numbers $k$ and $j$. The solution to the eigenvalue equation Eq $\overline{B 6}$ is now $\left(\left[\underline{\underline{V}}\left(t^{\prime \prime}\right)\right]_{\alpha, \alpha^{\prime}} \approx 0\right)$

$$
\chi_{j, k}\left(t^{\prime \prime}\right)=e^{+\frac{i}{\hbar} \lambda_{k} t^{\prime \prime}} e^{-\frac{i}{\hbar} \int^{t^{\prime \prime}} d \tau E_{j}^{a d}(\tau)} .
$$


By using Eq 30155156 we get that the adiabatic solution, in the (t,t',t") formalism, is given by,

$$
\widetilde{\Psi}^{R}\left(\mathbf{r}, t^{\prime}, t^{\prime \prime}, t\right)=\left[\sum_{k} c_{k} e^{-\frac{i}{\hbar} \lambda_{k}\left(t-t^{\prime \prime}\right)}\right] \phi_{j}^{a d, R}\left(\mathbf{r}, t^{\prime}, t^{\prime \prime}\right) e^{-\frac{i}{\hbar} \int^{t^{\prime \prime}} d \tau E_{j}^{a d}(\tau)}
$$

Now, applying the cut $t^{\prime \prime}=t$ in order to obtain the physical solution of Eq22 one gets (eliminating the phase factor $\sum_{k} c_{k}$ ):

$$
\Psi^{R}(\mathbf{r}, t)=\left.\widetilde{\Psi}^{R}\left(\mathbf{r}, t^{\prime}, t^{\prime \prime}, t\right)\right|_{t^{\prime \prime}=t^{\prime}=t}=\left.e^{-\frac{i}{\hbar} \int^{t} d \tau E_{j}^{a d}(\tau)} \phi_{j}^{a d, R}\left(\mathbf{r}, t^{\prime}, t^{\prime \prime}\right)\right|_{t^{\prime \prime}=t^{\prime}=t}
$$

This is the adiabatic solution of the TDSE associated with initial state $\varphi_{j}(\mathbf{r})$.

Let us summarize and clarify the procedure that need to be made in order to determine if a pulse is adiabatic or not; The determination is carried out through the calculation of the expression $A_{(j, 0)}(t)$ (it is t now, not t" !):

(1) Perform non-hermitian adiabatic Floquet simulations (Eq 34) with CW field, for a range of intensities $\varepsilon_{1}$ which covers all intensities between zero and the maximal intensity $\varepsilon_{0}$ that the studied laser pulse reaches. The adiabatic Floquet Hamiltonian is therefore

$$
H_{a d}\left(\mathbf{r}, t^{\prime}, \varepsilon_{1}\right) \equiv H_{0}(\mathbf{r})-e \mathbf{r} \cdot \varepsilon_{1} \cos \left(\omega t^{\prime}\right)-i \hbar \frac{\partial}{\partial t^{\prime}}
$$

and the eigenvalue equation to be solved is

$$
H_{a d}\left(\mathbf{r}, t^{\prime}, \varepsilon_{1}\right) \phi_{j}^{a d, R}\left(\mathbf{r}, t^{\prime}, \varepsilon_{1}\right)=E_{j}^{a d}\left(\varepsilon_{1}\right) \phi_{j}^{a d, R}\left(\mathbf{r}, t^{\prime}, \varepsilon_{1}\right)
$$

Obtain the quasienergy spectrum $E_{j}^{a d}\left(\varepsilon_{1}\right)$ and all the adiabatic cross terms $c_{\left(j^{\prime}, m\right)}^{(j, 0)}\left(\varepsilon_{1}\right)$ as defined in Eq 5360 as function of the intensities. This stage is done only once, for a given system.

(2) For a given laser pulse $f(t)$ with a maximal intensity $\varepsilon_{0}$, evaluate the effective CW-field intensity as function of time $\varepsilon_{0} f(t)(\mathrm{Eq}$ [24). Then, convert the adiabatic cross terms to be functions of time via the equality $(\mathrm{Eq} 33$ )

$$
\varepsilon_{1}=\varepsilon_{0} f(t) .
$$

using $c_{\left(j^{\prime}, m\right)}^{(j, 0)}\left(\varepsilon_{1}\right)$ as calculated in step $1 c_{\left(j^{\prime}, m\right)}^{(j, 0)}(t)=c_{\left(j^{\prime}, m\right)}^{(j, 0)}\left(f^{-1}\left(\varepsilon_{1} / \varepsilon_{0}\right)\right)$ where $f^{-1}$ is the transformation which fulfills $f^{-1}[f(t)]=t$.

(3) For each given resonance state $\alpha=(j, 0)$ calculate

$$
A_{(j, 0)}(t)=F(t) \cdot \sum_{j^{\prime} \neq j} \sum_{m \neq 0}\left|c_{\left(j^{\prime}, m\right)}^{(j, 0)}(t)\right|
$$

using $c_{\left(j^{\prime}, m\right)}^{(j, 0)}(t)$ from step 2 where notice that here $t^{\prime \prime}$ in Eqs. (50) 531) is replaced by $t$. If for a given resonance state $\alpha=(j, 0)$ the corresponding expression $A_{(j, 0)}(t)$ is smaller then unity at every instant, it is guaranteed that the system 
initially at the bound state which corresponds to this resonance, will evolve adiabatically to that resonance. In this case the HGS spectra will show only odd harmonics and the ionization probability as function of time will have a simple form that will be shown.

\section{Illustrative numerical example}

We studied a single-electron 1D Xe atom subjected to a single sin-square pulse of strong monochromatic laser field in two approaches. In the first one hermitian simulations were carried out whereas in the second non-hermitian Floquet simulations based on the complex scaling method were carried out. The hermitian simulations were carried out by solving the following TDSE

$$
\left[-\frac{\hbar^{2}}{2 m} \frac{\partial^{2}}{\partial x^{2}}+V_{0}(x)-e x \varepsilon_{0} f(t) \cos (\omega t)\right] \Psi(x, t)=i \hbar \frac{\partial}{\partial t} \Psi(x, t)
$$

with a sine-square envelope

$$
f(t)=\sin \left(\frac{\omega t}{2 N}\right)
$$

and the field-free effective potential $V_{0}(x)$ was an inverse Gaussian

$$
V_{0}(x)=-0.63 \exp \left(-0.1424 x^{2}\right)
$$

which supports two bound states that mimic the two lowest electronic states of Xe, with energies $E_{0}=-0.4451 a . u ., E_{1}=-0.1400 a . u$. and a third weakly bound state with energy $E_{2}=-0.00014 a . u$.

The wave function was taken initially at the ground state (g.s.) of the fieldfree Hamiltonian of the system:

$$
\Psi(x, t=0)=\varphi_{1}(x)
$$

and was calculated for times $0<t<N T$ (single sine-square pulse, $N$ was the number of optical cycles that entered the pulse). Using this wavefunction the ionization probability at times $t=\frac{\pi}{2 \omega}+n \frac{\pi}{\omega}, 0 \leq n \leq 2 N-1$ was calculated (at these times the potential felt by the electron was the field-free potential $V_{0}(x)$ )

$$
P_{\text {ion }}(t)=1-\sum_{i=1}^{3}\left|\left\langle\varphi_{i} \mid \Psi(t)\right\rangle\right|^{2}
$$

where $\varphi_{i}$ were the bound states of the field-free problem (3 bound states over which we summed in this example). Also HGS was calculated, which following the classical-quantum correspondence principle (Larmor formula, 24]) equals the modulus-square of the Fourier-transformed time-dependent acceleration expectation value. This is actually the intensity of the radiation emitted by the oscillating electron as presented in energy space. 


$$
\sigma(\Omega)=\left|\frac{1}{N T} \int_{0}^{N T} \frac{1}{m}\left\langle\Psi(t)\left|-\frac{\partial V_{0}(x)}{\partial x}+e \varepsilon_{0} f(t) \cos (\omega t)\right| \Psi(t)\right\rangle e^{-i \Omega t} d t\right|^{2}
$$

The non-hermitian simulations were Floquet simulations which were carried out for different field intensities. The quasienergy spectrum of complex energies $E_{j^{\prime}}^{a d}\left(\varepsilon_{1}\right)$ and the adiabatic cross terms $c_{\left(j^{\prime}, m\right)}^{(j, 0)}\left(\varepsilon_{1}\right)$ were calculated for each intensity. Then, these quantities were expressed as function of time through Eq 33 i.e. $t=f^{-1}\left(\varepsilon_{1} / \varepsilon_{0}\right)$. It was verified that in the cases where the adiabatic criteria was fulfilled, the HGS obtained from the hermitian propagation simulation contained only odd harmonics. A more quantitative measure of the existence of the adiabatic criteria was obtained through the comparison of ionization probabilities as obtained from the hermitian propagation simulation and the non hermitian simulation.

In order to get non-hermitian Floquet Hamiltonian, the complex coordinate method was used. The Floquet Hamiltonian (Eq[59)

$$
H_{a d}\left(x, t^{\prime}, \varepsilon_{1}\right) \equiv-e^{-2 i \theta} \frac{\hbar^{2}}{2 m} \frac{\partial^{2}}{\partial x^{2}}+V_{0}\left(x e^{i \theta}\right)-e x e^{i \theta} \varepsilon_{0} f(t) \cos (\omega t)-i \hbar \frac{\partial}{\partial t^{\prime}}
$$

was diagonalized. Provided that the scaling parameter $\theta$ was sufficiently large, the resonance quasienergy states were $\theta$ independent:

$$
E_{j}^{a d}\left(t^{\prime \prime}\right)=E_{r_{j}}\left(t^{\prime \prime}\right)-\frac{i}{2} \Gamma_{j}\left(t^{\prime \prime}\right) ; \quad \Gamma_{j}=\frac{\hbar}{\tau_{j}}
$$

$E_{r_{j}}$ being the position of the state, $\Gamma_{j}$ being the width of the state and $\tau_{j}$ its lifetime. Since the resonance states had finite lifetimes, and since the resonances are the states which are associated with the dynamics, these resonance lifetimes should have fingerprints in the hermitian propagation simulation. Indeed it was found to be so when the ionization probabilities, as computed in the two simulations, were compared; The ionization probability at each instant was given by the following expression, which was obtained using Eq 58 and the F-product definition for the inner product [13]:

$$
P_{\text {ion }}(t)=e^{-\frac{1}{\hbar} \int^{t} d \tau \Gamma_{1}(\tau)}
$$

where $\Gamma_{1}(\tau)$ was associated with the Floquet resonanace state that was "born" from the ground stationary state $\varphi_{1}(\mathbf{r})$ as the field was turned on. The resonances that were "born" from the field-free Hamiltonian bound states were identified by plotting the quasienergy spectrum as function of the effective field intensity $\varepsilon_{1}$. The resonance complex quasienergy trajectories started from the field-free Hamiltonian bound states real energies and formed continuous trajectories in the complex energy plane as function of the intensity.

How was this expression for the ionization probability obtained? According to the F-product definition when the complex energy given in Eq69 is 
substituted in Eq[58 the "ket" (right) solution of the non-hermitian TDSE is obtained:

$$
\Psi^{R}(\mathbf{r}, t)=\left.e^{-\frac{1}{2 \hbar} \int^{t} d \tau \Gamma_{j}(\tau)-\frac{i}{\hbar} \int^{t} d \tau E_{r_{j}}(\tau)} \phi_{j}^{a d, R}\left(\mathbf{r}, t^{\prime}, t^{\prime \prime}\right)\right|_{t^{\prime \prime}=t^{\prime}=t}
$$

and it is easily seen that this function decays with time. The "bra" (left) solution is not a solution of a Schrödinger equation but is derived from the "ket" solution (the explanation of this point is beyond the scope of this work; for an explanation see [14])

$$
\Psi^{L}(\mathbf{r}, t)=\left.e^{-\frac{1}{2 \hbar} \int^{t} d \tau \Gamma_{j}(\tau)+\frac{i}{\hbar} \int^{t} d \tau E_{r_{j}}(\tau)} \phi_{j}^{a d, L}\left(\mathbf{r}, t^{\prime}, t^{\prime \prime}\right)\right|_{t^{\prime \prime}=t^{\prime}=t}
$$

and also this function decays with time. When the F-product of $\Psi^{L}(\mathbf{r}, t)$ and $\Psi^{R}(\mathbf{r}, t)$ is calculated (this is an overlap integral without complex conjugation of the left state; in the usual dirac-product notation this reads $\left.\left\langle\Psi^{L *}(t) \mid \Psi^{R}(t)\right\rangle\right)$ the terms containing the real part of the energy cancel each other and the overlap integral (c-product) of the adiabatic Floquet states gives unity (remember the completeness property of Floquet states also in coordinate space alone). The expression in Eq[70 is obtained for the specific case that only the Floquet resonance state which is "born" from the field-free bound state is populated. When several Floquet states are populated the same type of calculation could be repeated, where this time $\Psi^{L}(\mathbf{r}, t)$ and $\Psi^{R}(\mathbf{r}, t)$ are given by liner combination of terms as in Eq171 with the proper coefficients. Since the adiabatic Floquet states are orthogonal to each other, a generalization of the result of Eq[70] is obtained:

$$
P_{\text {ion }}(t)=\sum_{i=1}^{3}\left|\left\langle\varphi_{i} \mid \Psi(t=0)\right\rangle\right|^{2} e^{-\frac{1}{\hbar} \int^{t} d \tau \Gamma_{i}(\tau)}
$$

Notice that in the non-hermitian formalism the norm is not conserved but decays with time; it contains the knowledge about decay processes inherently.

The two functions as given in Eq 66 w0 were compared and it was found that the resemblance between the functions increased as the adiabatic limit was increasingly reached by the laser pulse parameters.

The numerical method used to solve the TDSE with hermitian Hamiltonian was the split operator Forest-Ruth algorithm with 7 points [25]; The grid size, time step and/or grid step were adjusted as required to achieve convergence. For the non-hermitian Floquet simulation, the (t,t') formalism was used, with the complex coordinate method. The number of basis functions, box length and scaling angle were adjusted as required to achieve convergence.

In Fig प the HGS as obtained for pulse strength of $\varepsilon_{0}=0.035 a . u$. (corresponding to intensity of $4.30 \cdot 10^{13} \mathrm{~W} / \mathrm{cm}^{2}$ ), pulse-durations of $N=5,10,50 \mathrm{opti}$ cal cycles, laser frequencies of $\omega=0.015 a . u$., 0.07a.u., 0.11a.u. (corresponding to energies of $\hbar \omega=0.408 \mathrm{eV}, 1.90 \mathrm{eV}, 2.99 \mathrm{eV}$ and wavelengths of $\lambda=3040 \mathrm{~nm}, 651 \mathrm{~nm}$, $414 n m$, respectively) and initial state $\varphi_{1}(x)$ are shown. The appearance of odd harmonics and the absence of even or non-integer harmonics, is in general the main feature which appears for long pulses, regardless of the frequency. The odd 
harmonics are obtained even for the shortest laser pulses although the existence of odd-symmetry selection rules is sensitive to the frequency: some deviation appears at frequency of $\omega=0.07 a . u$. and the obtained spectra is more complicated.

In Fig 2 the complex quasienergies of the 3 resonances which are "born" from the 3 bound states of the field-free Hamiltonian (will be given indices 13 from now on), as obtained from adiabatic Floquet simulations (Eq 60) for $\omega=0.11 a . u$. are shown as function of the $\mathrm{CW}$-field strength $\varepsilon_{1}$. Notice that the lifetimes of these resonances are not monotonic functions of the field intensity. It should be noted that these were not the only resonances that appeared in the quasienergy spectrum; there were also other resonances which didn't emerge from the field-free bound states. However, as will be shown in Fig 4 in this case for not too large field intensities resonances 1-3 were the only important resonances and they alone determined the dynamical behavior of the system.

In Fig [3 the ionization probability as obtained from the hermitian simulation (Eq 66) with pulse strength of $\varepsilon_{0}=0.035 a . u$., pulse-durations of $N=5,10,50$ optical cycles, laser frequency of $\omega=0.11 a . u$. and initial state $\varphi_{1}(x)$, is compared to the ionization probability as obtained from the expression given in Eq[70] which is derived from the F-product formalism together with the resonances quasienergies obtained from the non-hermitian simulation. It is seen that as the pulse becomes longer the results obtained from the two simulations become identical.

Both results of HGS and ionization probabilities showed that the timedependent wavefunction of the studied systems could be well approximated by the adiabatic expression given in Eq[58] even for short pulses. The values of the terms which check the adiabatic criteria, as seen in Fig [ gave the explanation why this was so.

In Fig团 the expressions $a_{(2)}^{(1)}, a_{(3)}^{(1)}, a_{(3)}^{(2)}(\mathrm{Eq} \sqrt[52]{52})$ which describe the couplings between every two resonances from the set of 3 tracked resonances is shown as function of the CW-field strength $\varepsilon_{1}$, for the case $\omega=0.11 a$.u. It can be seen that the coupling between resonances 1 and 2 is strong for field strength of $\varepsilon_{1} \simeq 0.04 a . u$., 0.064a.u. and 0.072a.u.. This could be partially explained on the basis of the values of the quasienergies, as seen in Fig 2 at least for two field strengths out of the three. It is seen that for field strength of 0.04a.u. and 0.064a.u. the real parts of the quasienergies cross, resulting in small value of the denominator in the expression given in $\mathrm{Eq} 53]$ for $c_{(2, m)}^{(1,0)}$ (at least for one $m$ term). In the same way the strong couplings between resonances 1 and 3 at field strength of $\varepsilon_{1}=0.064 a . u$., and between resonances 2 and 3 at field strength of $\varepsilon_{1}=0.066 a$.u. could be explained on the basis of the quasienergy values at these field-strengths. In particular it should be noticed that the couplings between resonances 1 and 3 at $\varepsilon_{1}=0.064 a$.u. are the strongest among all 3 resonances due to the close values of both real and imaginary parts of the quasienergies. However, it should be noted that crossings in the quaisenergy plot are not always indications of large couplings since also the overlap between the wavefunctions (the nominator of the expression for $c_{\left(j^{\prime}, m\right)}^{(j, 0)}$ ) is important. 
In Fig 5 the sum $\sum_{j^{\prime} \neq 1} a_{\left(j^{\prime}\right)}^{(1)}\left(\varepsilon_{1}\right)$ which describes the couplings between all quasistates of the system $\alpha^{\prime}=\left(j^{\prime}, m\right)$ to resonance $1 \alpha=(1,0)$ is shown as function of the CW-field strength $\varepsilon_{1}$, for the case $\omega=0.11 a$.u.. In addition, also the partial sum $a_{(2)}^{(1)}\left(\varepsilon_{1}\right)+a_{(3)}^{(1)}\left(\varepsilon_{1}\right)$ which describes the couplings between resonances 2 and 3 to the resonance state $\alpha=(1,0)$ is shown. It can be shown that up to a moderate intensity of $\varepsilon_{1} \sim 0.04 a$.u. the first resonance is mainly coupled only to the other 2 resonances and not to other, higher resonances or continuum states. The entire dynamics is governed almost solely by the 3 resonances which are "born" from the 3 bound states of the field-free Hamiltonian.

The sums described above, which are functions of a CW-field strength $\varepsilon_{1}$, are converted to be explicit functions of time for the specific sine-square pulse with maximal intensity $\varepsilon_{0}=0.035 a$.u . used in the simulation (Fig [ 5 upper part). For this purpose for each time $t 0<t<N T$ the effective CW-field strength $0.035 f(t)$ is calculated and the values of the 2 functions shown in Fig which fit this effective CW-field strength are taken. Hence the 2 sum functions are converted to be explicit functions of time. In the middle part of Fig [6 the full term $A_{(1,0)}(t)$, which represents the degree of adiabaticity in the process of shining a $1 \mathrm{D}$ Xe atom initially at the ground state with a sine-square laser pulse supporting $N=5$ optical cycles of monochromatic radiation with frequency $\omega=0.11 a$.u. and strength $\varepsilon_{0}=0.035 a$.u., is shown as function of time. It is seen that the term $A_{(1,0)}(t)$ is bounded by the value of $\sim 2 \cdot 10^{-3}$ for all times, whether it is calculated by coupling of the first resonance to other 2 resonances only or to all other states. The structure of the HHS for $N=5$ seen in Fig 1 implies that this value is indeed small and the process is adiabatic. In the lower part of Fig [ 6 the same quantity is shown, but for $\omega=0.07 a . u$.. Here it is seen the terms $A_{(1,0)}(t)$ are bounded by a much larger value of $\sim 10^{-1}$ for all times and the more complex structure of the HHS for $N=5$ seen in Fig पimplies that this value is not small enough to indicate the appearance of an adiabatic process. It should be noted that for a given system with field-strength $\varepsilon_{0}$, frequency $\omega$ and sine-square pulse envelope, we have $F(t)=|e|\left(\frac{\hbar \varepsilon_{0} \omega}{2 N}\right)\left|\sin \left(\frac{\omega t}{N}\right)\right|$. Therefore, as the number of optical cycles the pulse supports increases the general shape of the terms $A_{(1,0)}(t)$ is kept the same but is attenuated. Therefore, for $\omega=0.07 a$.u. as $N$ gets bigger, the pulse's envelope varies more slowly and the process becomes more and more adiabatic, as seen in the HHS spectra for $N=50$ for example.

\section{Conclusions}

With the help of the $\left(t, t^{\prime}\right)$ formalism we derive an adiabatic theorem for open systems. The use of the complex scaling transformation plays a key role in our derivation. For example, the spectrum of the Floquet Hamiltonian of an open system is changed dramatically. Rather than a continuous spectrum that is responsible for the absence of an adiabatic limit for $N$ (number of basis functions $) \rightarrow \infty$ in the conventional QM, the resonances are associated with a point spectrum and are separated from the continuum which is rotated into the lower half of the complex energy plane. 
An interesting important numerical result of our derivation is that the calculation of the effect of the pulses's shape on the dynamics does not require heavy computations. The entire effect of the laser pulse is embedded in a multiplication factor of $d f(t) / d t$ where $\varepsilon_{0} f(t)$ is the variation of the maximum field amplitude as function of time.

As a numerical example we applied the adiabatic theorem we derived to a model Hamiltonian of Xe atom (with symmetric field-free potential) which interacts with strong, monochromatic laser pulses. We have shown that the generation of odd-order harmonics and the absence of even-order harmonics, even when the pulses are extremely short, can be explained with the help of the adiabatic theorem we derived.

The use of a single-electron 1D model to describe a realistic atom is justified since it has been shown before in many cases that all the main strong field effects are reproduced. Therefore the conclusions obtained with this model are also valid for a realistic atom.

\section{Acknowledgements}

This work was supported in part by the by the Israel Science Foundation and by the Fund of promotion of research at the Technion. Dr. Milan Šindelka is acknowledged for most helpful and fruitful discussions. 


\section{References}

[1] S. X. Hu and Z. Z. Xu, Phys. Rev. A 56, 3916 (1997); S. Dionissopoulou, T. Mercouris and C. A. Nicolaides, ibid. 61, 063402 (2000); A. Di Piazza and E. Fiordilino, ibid. 64, 013802 (2001); V. Véniard, R. Tä̈eb and A. Maquet, ibid. 65, 013202 (2001).

[2] T. Millack and A. Maquet, J. Mod. Opt. 40, 2161 (1993); N. Hay, R. de Nalda, T. Halfmann, K. J. Mendham, M. B. Mason, M. Castillejo and J. P. Marangos, Eur. Phys. J. D. 14, 231 (2001).

[3] N. Ben-Tal, N. Moiseyev and A. Beswick, J. Phys. B 26, 3017 (1993).

[4] O. E. Alon, V. Averbukh and N. Moiseyev, Phys. Rev. Lett. 80, 3743 (1998); O. E. Alon, V. Averbukh and N. Moiseyev, Phys. Rev. Lett. 85, $5218(2000)$.

[5] N. Ben-Tal, N. Moiseyev, C. Leforestier and R. Kosloff, J. Chem. Phys. 94, 1636-1642 (1991).

[6] N. V. Vitanov, T. Halfmann, B. W. Shore and K. Bergmann, Annu. Rev. Phys. Chem. 52, 763-809 (2001).

[7] U. Peskin and N. Moiseyev, J. C. P 99, 4590 (1993).

[8] A. Fleischer,V. Averbukh and N. Moiseyev, Phys. Rev. A, 69, 043404/1 (2004).

[9] W. P. Reinhardt, Annu. Rev. Phys. chem. 33, 223 (1982).

[10] N. Moiseyev, Physics Reports 302, 211-293 (1998).

[11] J. H. Wilkinson, "The Algebraic Eigenvalue Problem", Clarendon Press, Oxford, 1965.

[12] N. Moiseyev, P.R. Certain and F. Weinhold, Mol. Phys., 36, 1613-1630 (1978).

[13] N. Moiseyev and M. Lein, J. Phys. Chem., 107, 7181 (2003).

[14] I. Gilary, A. Fleischer and N. Moiseyev, submitted for publication.

[15] D. W. Hone, R. Ketzmerik and W. Kohn, Phys. Rev. A 56, 4045 (1997).

[16] K. Drese and M. Holthaus, Eur. Phys. J. D. 5, 119 (1999).

[17] D. Barash, A. E. Orel and R. Baer, Phys. Rev. A 61, 013402 (1999).

[18] S. I. Chu and W. P. Reinhardt, Phys. Rev. Lett. 39, 1195 (1977).

[19] M. Dörr, O. Latinne and C. J. Joachain, Phys. Rev. A, 55, 3697 (1997);

[20] H. C. Baker, Phys. Rev. A, 30, 773 (1984); 
[21] R. M. Potvliege and R. Shakeshaft, Phys. Rev. A, 38, 4597 (1988).

[22] H. C. Day, B. Piraux and R. M. Potvliege, Phys. Rev. A, 61, 031402(R) (2000);

[23] J. Aguilar and J. M. Combes, Commun. Math. Phys. 22, 269 (1971); J. Balslev and J. M. Combes, Commun. Math. Phys. 22, 280 (1971); B. Simon, Commun. Math. Phys. 27, 1 (1972).

[24] L. D. Landau and E. M. Lifshitz, "Classical Theory of Fields" (Butterworth-Heinenann Press, oxford, 1975), 4th edition.

[25] J. A. Fleck, J. R. Morris and M. D. Feit, Appl. Phys. 10, 129 (1976); M. D. Feit, J. A. Fleck and A. Steiger, J. Comput. Phys. 47, 412 (1982); E. Forest and R. D. Ruth, Physica D 43, 105 (1990); M. Campostrini and P. Rossy, Nucl. Phys. B 329, 753 (1990); J. Candy and W. Rozmus, J. Comput. Phys. 92, 230 (1991). 


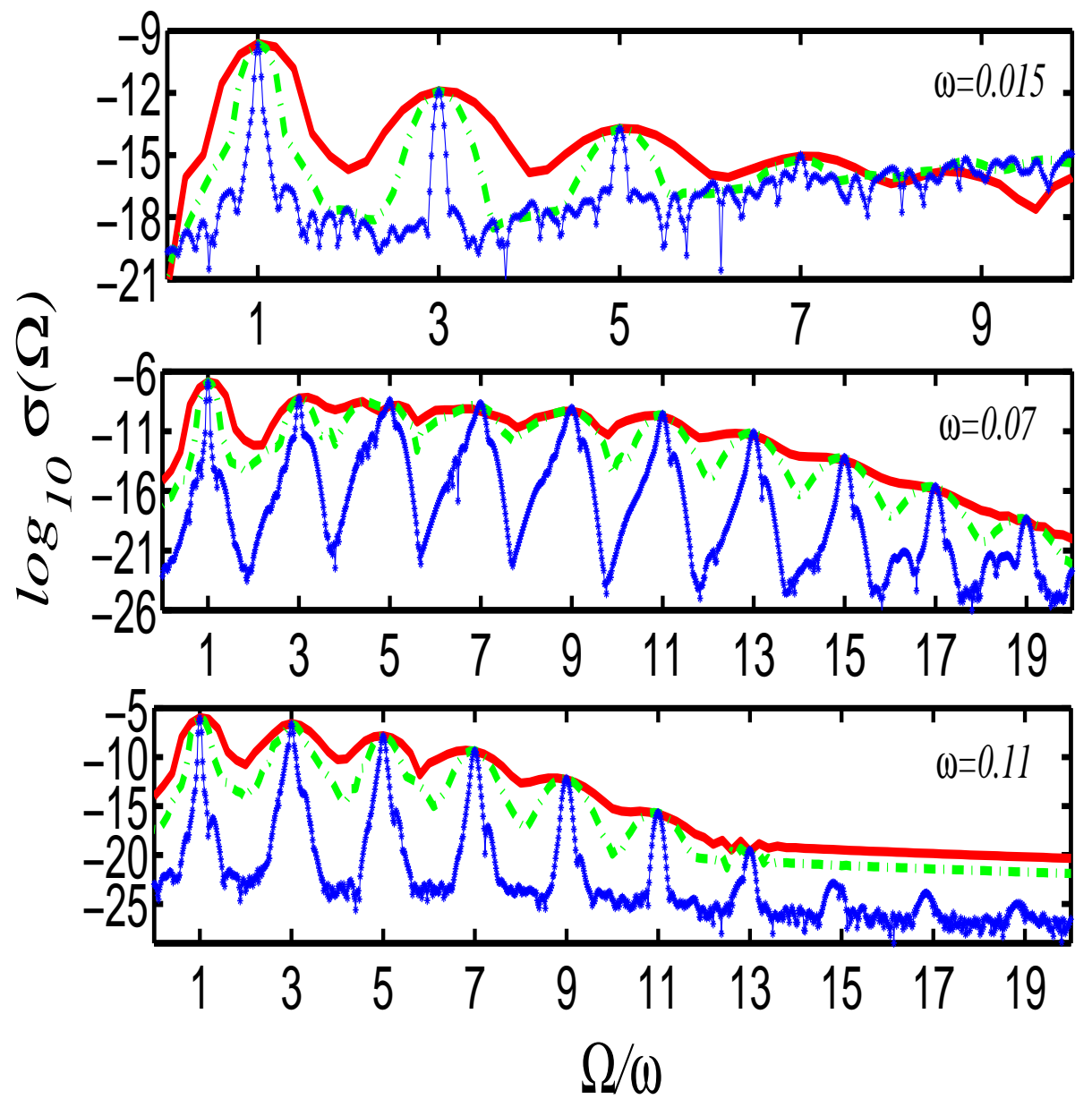

Figure 1: (color online) HGS obtained from hermitian simulations describing a $1 \mathrm{D}$ Xe atom subjected to various sin-square pulses with strength of $\varepsilon_{0}=$ $0.035 a . u$., laser frequencies of $\omega=0.015 a . u$. (upper part), $\omega=0.07 a . u$. (middle part) and $\omega=0.11 a . u$. (lower part) and pulse-durations of $N=5$ (solid), 10 (dot-dashed) and 50 (solid-dotted) optical cycles. The initial state was taken to be the ground state in all simulations. For the longest pulse duration only odd harmonics appear in the spectrum, and hyper Raman lines are absent. This is also the basic feature in the case of short pulses, although some deviation from this structure appear at frequency of $\omega=0.07 a . u$.. 

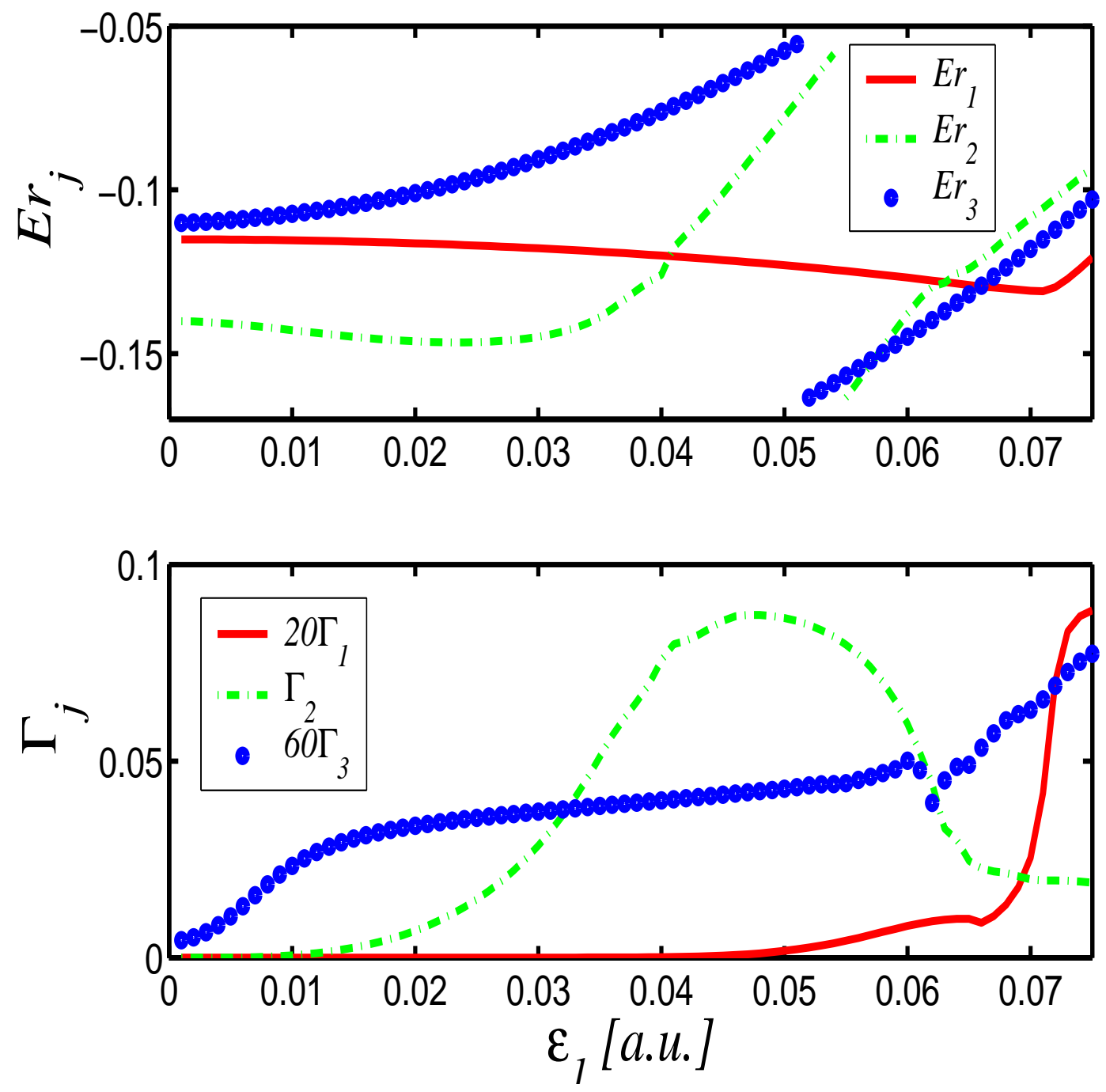

Figure 2: (color online) Positions (upper part) and lifetimes (lower part) of the complex quasienergies of resonances 1-3 as function of the $\mathrm{CW}$-field strength $\varepsilon_{1}$ as obtained from adiabatic non-hermitian Floquet simulations (Eq 60669) for $\omega=0.11 a . u$. 


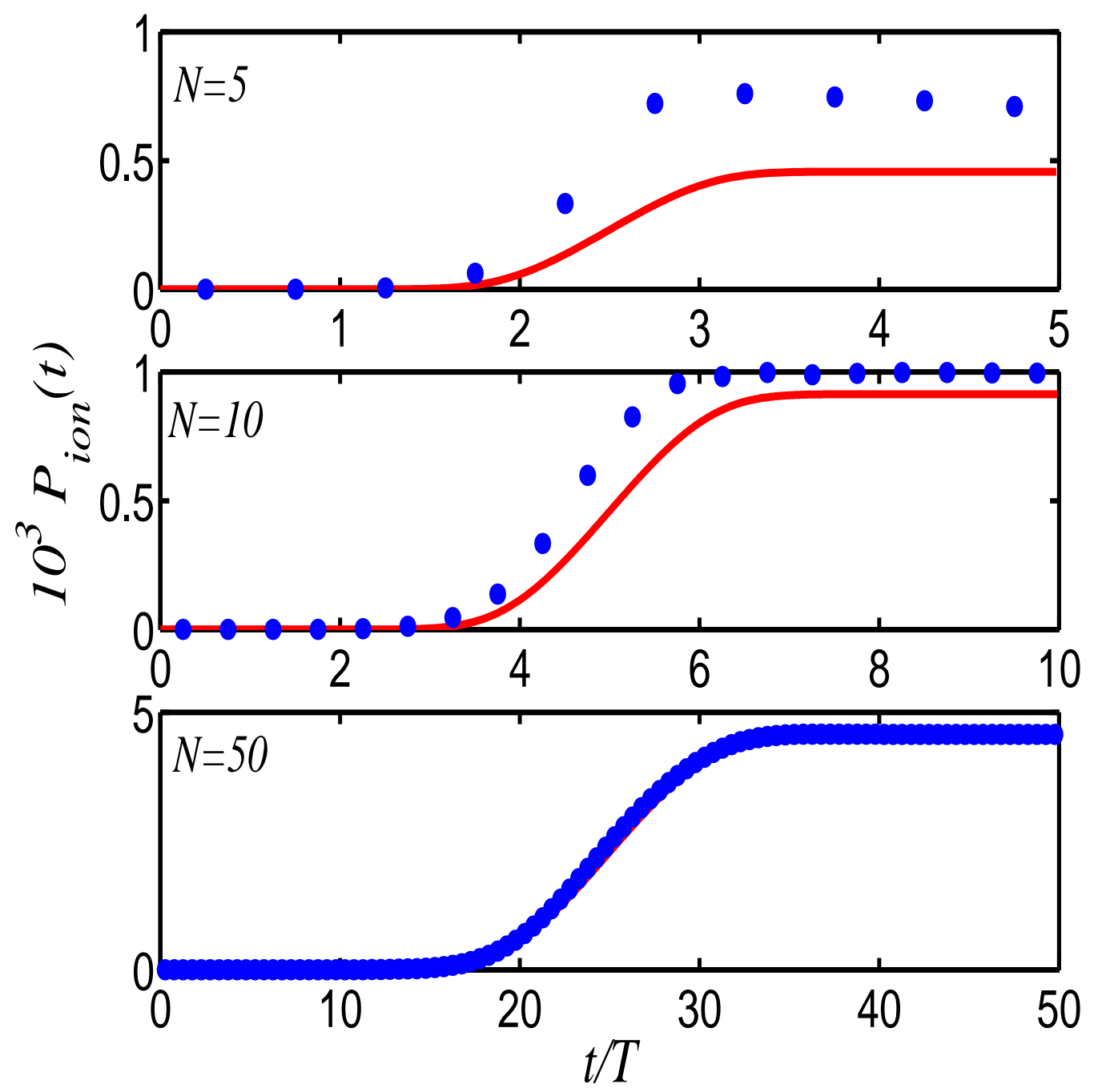

Figure 3: (color online) Ionization probabilities obtained from hermitian simulations (lines) (Eq 66) for pulse-durations of $N=5$ (upper part), $N=10$ (middle part), $N=50$ (lower part) optical cycles, and from non-hermitian simulations (dots) (Eq 701). As the pulse becomes longer the results obtained from the two simulations become identical since the adiabaticity of the process is increased. 


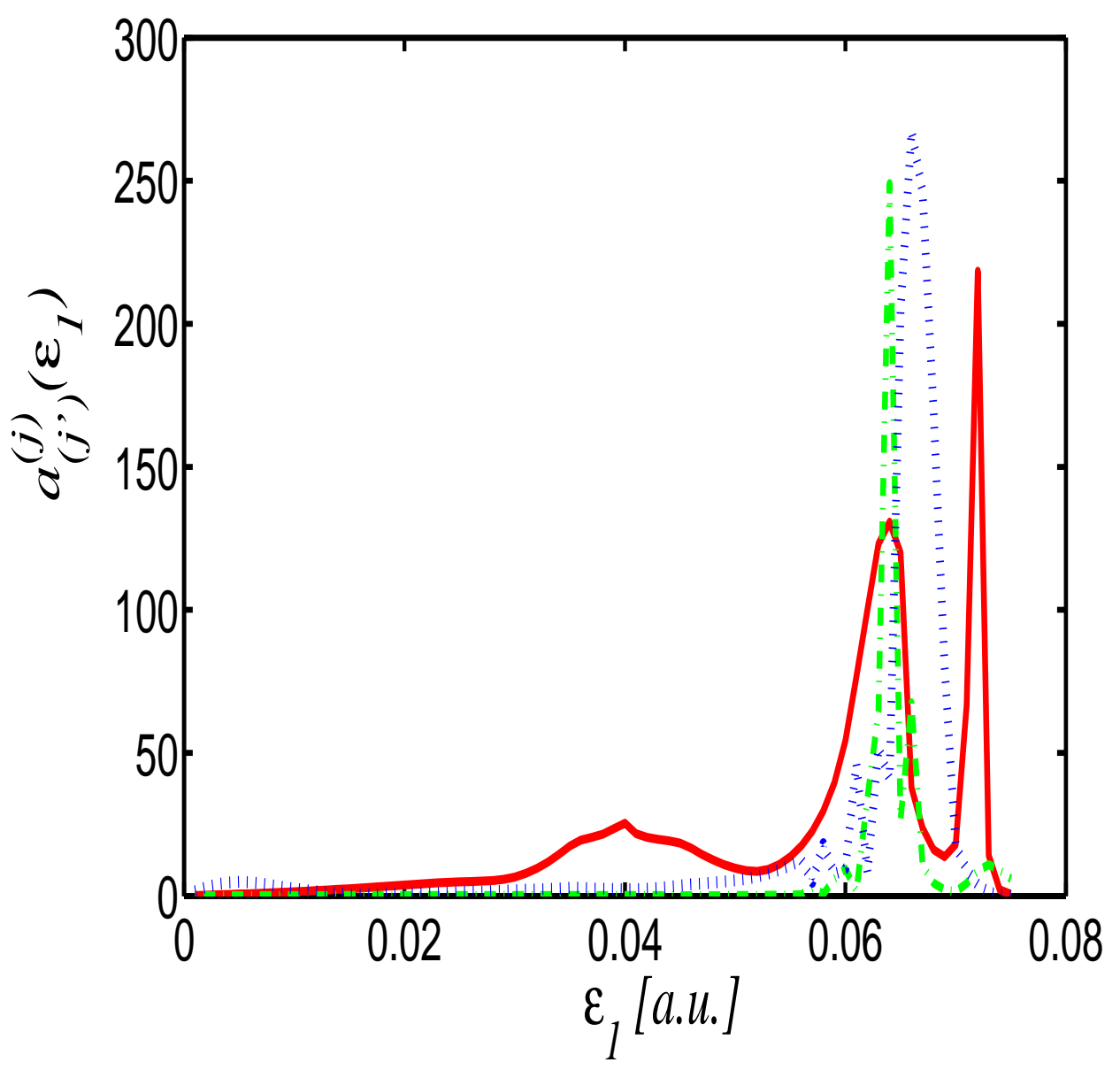

Figure 4: (color online) The expressions $6 a_{(2)}^{(1)}\left(\varepsilon_{1}\right)$ (solid), $a_{(3)}^{(1)}\left(\varepsilon_{1}\right)$ (dashed), $2.5 a_{(3)}^{(2)}\left(\varepsilon_{1}\right)$ (dotted) $\left(\mathrm{Eq}[52)\right.$ as function of the CW-field strength $\varepsilon_{1}$, for $\omega=$ 0.11a.u.. 


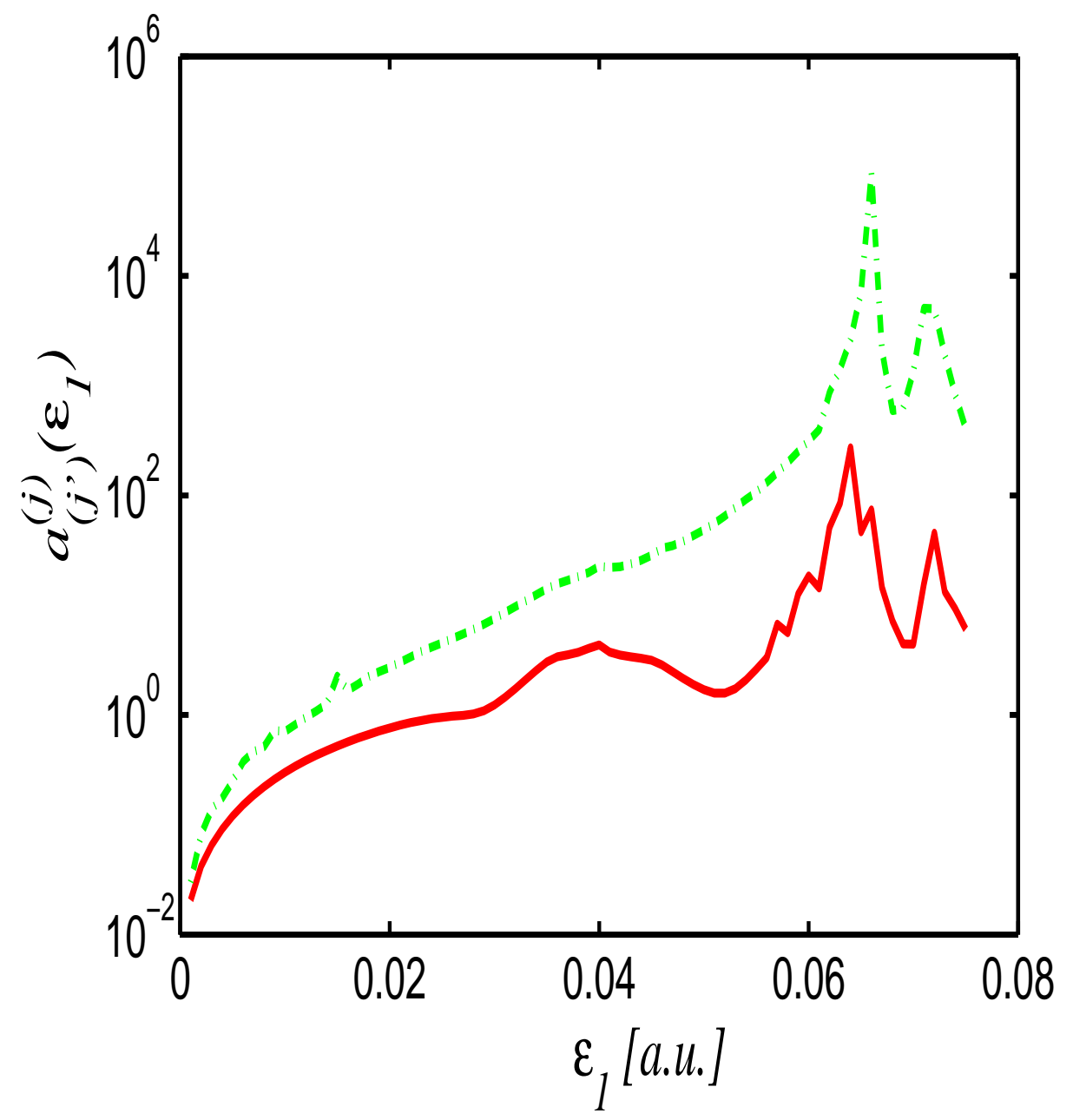

Figure 5: (color online) The expressions $\sum_{j^{\prime} \neq 1} a_{\left(j^{\prime}\right)}^{(1)}\left(\varepsilon_{1}\right)$ (dashed) and $a_{(2)}^{(1)}\left(\varepsilon_{1}\right)+$ $a_{(3)}^{(1)}\left(\varepsilon_{1}\right)$ (solid), which describe respectively the couplings between all quasistates of the system $\alpha^{\prime}=\left(j^{\prime}, m\right)$ or only resonances 2 and 3 to resonance 1 $\alpha=(1,0)$ are shown as function of the $\mathrm{CW}$-field strength $\varepsilon_{1}$, for the case $\omega=0.11 a . u$. 


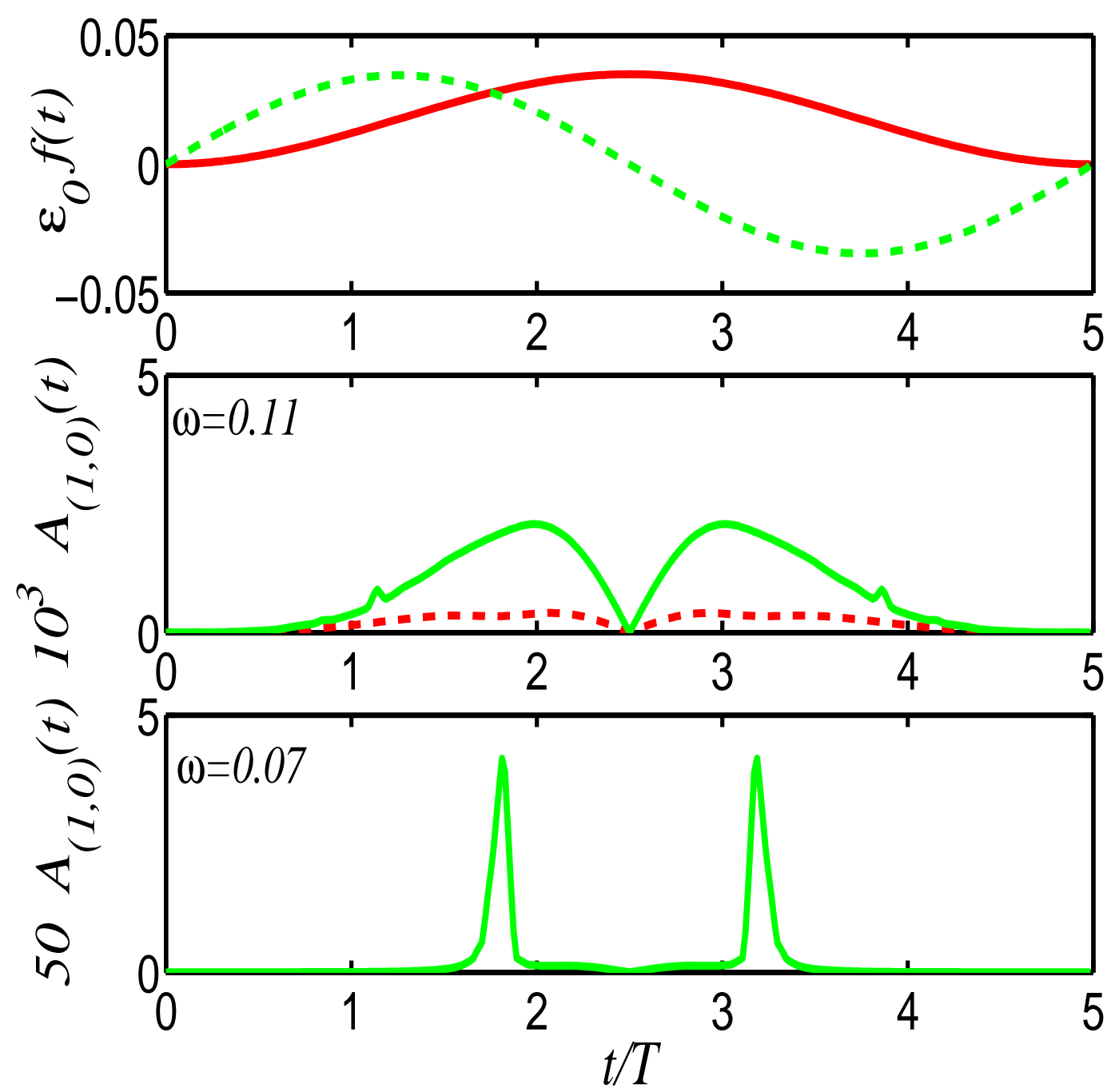

Figure 6: (color online) upper part: The time-dependence of a sine-square pulse $\varepsilon_{0} \sin ^{2}\left(\frac{\omega t}{2 N}\right)$ (solid) and a scaled time derivative $90\left(\frac{\varepsilon_{0} \omega}{2 N}\right) \sin \left(\frac{\omega t}{N}\right)$ (dashed) for $N=5, \omega=0.11$ a.u. and $\varepsilon_{0}=0.035$ a.u.. Middle part: the full term $A_{(1,0)}(t)$ (solid), and the partial term $F(t)\left(a_{(2)}^{(1)}(t)+a_{(3)}^{(1)}(t)\right.$ (dashed) are shown as function of time. Both terms are bounded by the small value of $\sim 2 \cdot 10^{-3}$ for all times. Lower part: the same as in the middle part, but for $\omega=0.07 a . u$.. Both terms are bounded by a larger value of $\sim 10^{-1}$. It is therefore deduced that for $\omega=0.11 a$.u. the process is adiabatic but for $\omega=0.07 a . u$. it is not and this is indeed verified in the HGS given in Fig 1 for $N=5$. 